\title{
Tracing Mechanism of Sports Competition Pressure Based on Backpropagation Neural Network
}

\author{
Huayu Zhao and Shaonan Liu (iD \\ Department of Sports, Northeastern University at Qinhuangdao, Qinhuangdao 066000, China \\ Correspondence should be addressed to Shaonan Liu; liushaonan@neuq.edu.cn
}

Received 10 December 2020; Revised 22 January 2021; Accepted 28 January 2021; Published 8 February 2021

Academic Editor: Wei Wang

Copyright (c) 2021 Huayu Zhao and Shaonan Liu. This is an open access article distributed under the Creative Commons Attribution License, which permits unrestricted use, distribution, and reproduction in any medium, provided the original work is properly cited.

\begin{abstract}
Through the overall situation of athletes' competition pressure, the pressure level of participating athletes can be understood and revealed. Analyzing the sources of stress and influencing factors of athletes can find measures to relieve and reduce stress and provide theoretical reference for the regulation of athletes' competition pressure. Based on genetic algorithm and neural network theory, this paper proposes a method of tracing the sports competition pressure based on genetic algorithm backpropagation (BP) neural network to solve the problem that traditional neural network learning algorithm is slow and easy to fall into local minimum. There is no significant difference between male and female athletes in the level of competition pressure. Athletes have the same training methods and the same goals, and the competition pressure tends to be the same, with no obvious difference; athletes with different educational backgrounds have no significant differences in training, academics, sports injuries, interpersonal relationships, social expectations, and evaluations. Due to the particularity of the stage, the competition pressure of fourth-year undergraduate and third-year masters is significantly higher than that of other grades. The number of athletes participating in college table tennis tournaments has very significant differences in competition dimensions. There is significant difference in training and self-expectation dimension difference. The competition pressure of athletes who participated in the college table tennis championship for the first time was significantly higher than that of athletes who participated repeatedly. There were significant differences between athletes before and after adapting to the venue. Before adapting to the venue, the competition pressure of athletes is generally greater. After adapting to the venue, the competition pressure of athletes has been relieved.
\end{abstract}

\section{Introduction}

One of the most difficult tasks for athletes in their entire sports career is to overcome adversity and achieve their best performance [1]. Mental skills training can help athletes complete this difficult task. Over the past thirty years, traditional psychological skill training based on cognitive behaviorism has been dominant [2-4]. In recent years, interventions based on mindfulness training have begun to emerge in the field of sports psychology due to their gentle methods and their focus on the task at hand to reduce the past or future entanglement with them. With empirical support, they have proved their effectiveness in sports practice [5].

Neural network is an active borderline interdisciplinary. The artificial neural network is relative to the biological neural network system in biology [6]. Its purpose is to use a certain simple mathematical model to describe the structure of the biological neural network. To a certain extent, the intelligent behavior of the quasibiological neural network solves the problem of intelligent information that cannot be handled by traditional algorithms $[7,8]$. It is the basis of massive information parallel processing and large-scale parallel computing. Neural network is both a highly linear dynamic system and a self-organizing adaptive system, which can describe the intelligent behavior of cognition, decision-making, and control [9]. Psychologist Mcculloch and mathematician Pitts proposed the mathematical model of formal neurons and studied the potential functions of interconnecting several basic elements based on neuron models [10]. Hinton and Sejnowshi used the concepts and methods of statistical physics to propose a Boltzman machine model and used simulated annealing technology in the 
learning process to ensure that the system is globally optimal [11]. Related scholars published a series of research results and methods [12-14]. Because of their outstanding work, they have provided a catalyst for the research of neuron networks, making this research and application enter the heyday [15]. Later, Kosko proposed two-way associative memory and adaptive two-way associative memory to provide effective methods for learning in noisy environments [16]. Among them, backpropagation (BP) neural network has become the most mature and widely used one among many neural networks. The genetic backpropagation artificial neural network formed by the combination of genetic algorithm and BP neural network has the advantages of both genetic algorithm and artificial neural network and has been widely used in fault diagnosis and pattern recognition in recent years [17]. Relevant scholars proposed an online learning method suitable for nonlinear tracing based on a feedforward neural network structure [18]. This method absorbs the advantages of the least square method and the traditional online BP algorithm. It has fast convergence speed and good tracking performance and is suitable for nonlinear tracking. Researchers use neural networks to trace the source of multivariate time series, propose a comprehensive traceability method combining multiple information, and use stock trading as an example to trace the development of China's stock market using confidence stock price theory [19]. It further explains the characteristics of neural network traceability methods from the perspective of information utilization. Relevant scholars proposed a method for short-term power system load tracing using distributed hierarchical artificial neural networks, analyzed the structural principles of distributed hierarchical neural networks, and discussed the advantages of using the algorithm and the realization of this neural network [20]. Related scholars have proposed a adaptive fuzzy neural network for chaotic sequence traceability [21]. Compared with the traditional adaptive fuzzy neural network, this adaptive fuzzy neural network greatly reduces the number of neural network nodes without affecting the traceability accuracy. At the same time, the neural network is learned by the BP algorithm based on chaotic dynamics, which improves the convergence speed and traceability accuracy. Using this TS fuzzy neural network and the corresponding BP learning algorithm, the Mackey-Glass chaotic time series is traced. Compared with the traditional TS fuzzy neural network, better results were obtained, which verified the effectiveness of the method. Relevant scholars have introduced artificial neural network technology to the complex nonlinearity of deep foundation pit system and the importance of multistep tracing of foundation pit engineering deformation [22-25]. Through the analysis of an example of multistep tracing of deformation of a deep foundation pit in soft soil, the reliability and practicability of recurrent neural network for multistep tracing of deformation of foundation pit are demonstrated.

This paper proposes a method for tracing the pressure of sports competitions based on the genetic backpropagation artificial neural network and analyzes the influence of the selection of the number of nodes in the hidden layer of the genetic backpropagation artificial neural network on the iterative calculation speed and diagnosis results. In this paper, the number of hidden layer nodes is 13 , the allowable error range is 0.12 , the number of network training is small, and the recognition accuracy is high. According to the results of the survey, the reasons for the competition pressure of the athletes are analyzed, so that the coaches can correctly understand the psychological state of the athletes, so that in daily teaching and training, the athletes can be targeted to train the antistress level of the athletes. This facilitates the subsequent management and regulation of competition pressure and strives to adopt a control strategy under the premise of understanding the sources of athletes' pressure to maintain it within an appropriate standard and find ways to avoid high competition pressure. This will greatly improve the athletes' ability to deal with competition pressure. Before the game, the coaches can formulate suitable decompression methods for individuals based on the usual competition pressure assessment in order to maintain a good competitive state.

The rest of this article is organized as follows. Section 2 discusses related theories and technologies. Section 3 establishes a genetic backpropagation artificial neural network model. In Section 4, the tracing experimental analysis of athletes' competition pressure and factors affecting competition pressure are carried out. Section 5 summarizes the full text.

\section{Related Theories and Technologies}

2.1. Artificial Neural Network. Neural network enables people to study the principles, models, and applications of neural networks on the basis of deepening their understanding of the nervous system. The human nervous system is composed of two types of neurons and glial cells. Neurons are composed of cell bodies, dendrites, and axons, as shown in Figure 1. Tens of thousands of neurons work together to form a neural network to complete basic functions.

An artificial neural network is a simulation of a biological system. Its information processing function is determined by the input and output characteristics (activation characteristics) of the units (neurons) of the network, the topology of the network (the way neurons are connected), and the size of the connection weight (it is determined by the connection strength between neurons) and neuron threshold (which can be regarded as a special connection weight).

The artificial neural network uses a simple data processing unit to simulate a neuron as a node of the network and uses weights to simulate the relationship between each neuron (that is, the strength of synaptic connection). The positive weight acts as an excitatory synapse, and the negative weight functions act as an inhibitory synapse. A node has several inputs, similar to the dendrites of nerve cells, which receive excitatory and inhibitory signals from several other neurons. The calculation processing unit performs a weighted summation of all input values and generates an output value through the internal conversion function of the 


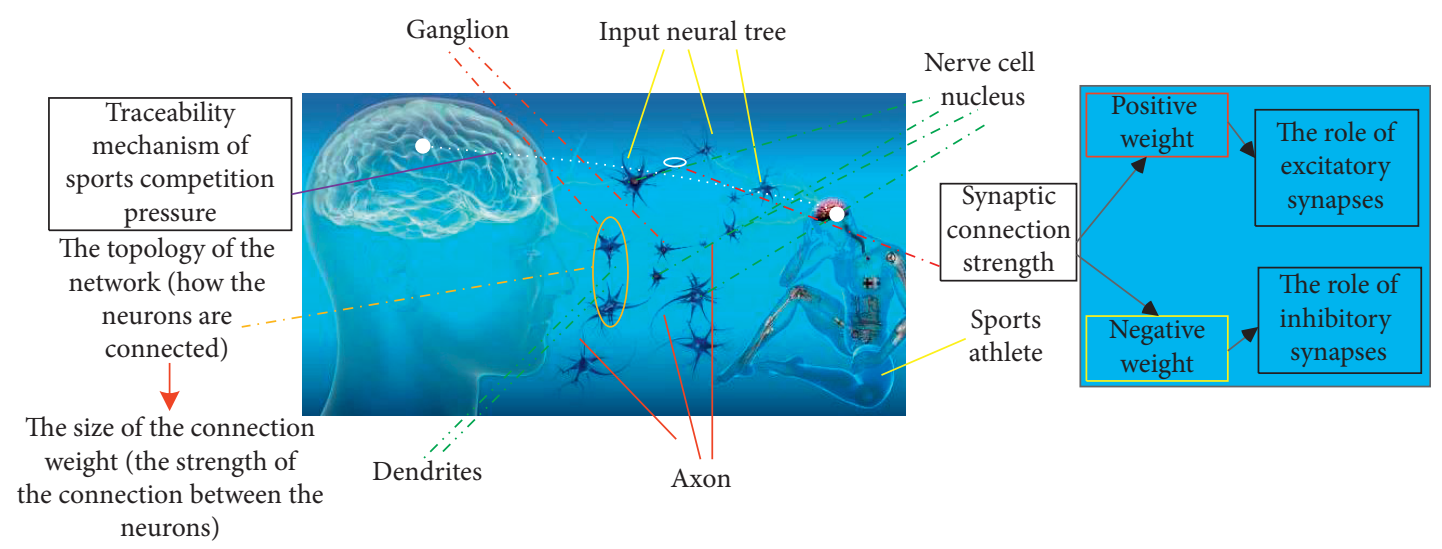

FIGURE 1: Schematic diagram of the mechanism of biological neurons.

weighted sum, whose function is equivalent to the axon that transmits the nerve impulse in the nerve cell.

2.2. Backpropagation Algorithm. The BP algorithm uses gradient search technology to minimize the mean square error between the output and the expected output. The learning process of the network is a process of propagating backward while correcting the weights. The principle and steps of the algorithm are described as follows:

In a multilayer network, there are two types of signals flowing:

(1) Working signal: it is the signal that propagates forward after applying the input signal until the actual output is produced at the output end. It is a function of the input vector and the weight.

(2) Error signal: the difference between the actual output signal of the network and the desired output is the error propagating backward from the output end layer by layer.

Suppose the input layer of the BP network has $m$ nodes, the hidden layer has $n$ nodes, and the output layer has $l$ nodes. The weight between the output layer and the hidden layer is $w_{i j}, i=1,2, n, j=1,2, m$, the weight between the hidden layer and the output layer is $t_{i j}$, and the activation function of the hidden layer unit and the output layer unit is the S-type sigmoid function, namely,

$$
f(x)=\frac{1}{\exp (-x)+1} .
$$

Suppose there is a training sample. The input vector of the sample is $X$ and the output vector is $Y$. Then, the input of each unit of the hidden layer is

$$
\text { Input }_{i}=\sum_{j=0}^{m-1} x_{j} \cdot\left(1-w_{i j}\right) .
$$

The output of each unit of the hidden layer is

$$
\text { Net }_{i}=\sum_{i=1}^{n} f\left(\text { Input }_{i}\right) .
$$

The input of each unit of the output layer is

$$
\text { Output }_{i}=\sum_{j=0}^{n-1} \mathrm{Net}_{j} \cdot t_{i j} \text {. }
$$

The output of each unit of the output layer is

$$
Y_{i}=\sum_{i=1}^{l} f\left(\text { Output }_{i}\right) .
$$

Suppose the ideal output of the $i$-th unit of the output terminal is $D_{i}$, then the error of this unit is

$$
E_{i}=D_{i}-Y_{i}
$$

Then, the total square error at the output is

$$
E=0.5 \cdot \sum_{i=1}^{l}\left(Y_{i}-D_{i}\right)^{2} \text {. }
$$

From the gradient descent method, the iterative equation of the network connection weight can be derived. The iterative equation of the connection weight between the input layer and the hidden layer is

$$
\Delta w_{i j}=\sum_{i=1}^{n} \sum_{j=1}^{m}\left(-\eta \frac{\partial E}{\partial w_{i j}}\right) .
$$

The iterative equation of the connection weight between the hidden layer and the output layer is

$$
\Delta t_{i j}=\sum_{i=1}^{l} \sum_{j=1}^{n}\left(-\eta \frac{\partial E}{\partial t_{i j}}\right) .
$$

\subsection{Descriptive Analysis of Competition Pressure}

2.3.1. Uncertainty. Regardless of the sport, competition pressure seems to be closely related to improving performance. It seems that competition pressure is also more influential in individual rather than team sports, but the coefficient of the relationship between competition pressure and performance is positive. When the athlete participates alone, the pressure is greater than the pressure when 
competing with other people in the team; when teammates are on the court, you may not be able to trace your performance because other players will affect the results. This result is similar to the results of other researchers. In many reports, the precompetitive pressure and performance traceability between teams and individual athletes are different. Later, sports were divided into open and closed skills. The relationship between competition pressure and performance has a stronger impact on open skills (that is, skills that are constantly changing in the environment) than on closed skills. It turns out that the uncertainty and importance of the results can explain how the competitive environment affects a person's stress state. Specifically, when an athlete is not sure whether he has the ability to obtain the desired result, and this result is important to the athlete, his or her stress level will increase.

2.3.2. Self-Confidence Conditions. Among the many influencing factors, self-confidence seems to affect whether the impact of the experienced competition pressure on performance is promoting or weakening. Confidence seems to regulate the impact of stress on performance. The same activity may produce stress in some people and happiness in others, and the level of confidence may be the experience mediator of stress or happiness. A neutral evaluation method based on the mixed effect of pressure on performance can replace competition pressure. Confidence and stress should be checked collectively. Stress and self-confidence are related constructs, and when athletes notice one of them, they may notice the other. By understanding the level of confidence and stress of athletes before the game, coaches and other sports professionals may be able to better trace the athlete's performance. In addition, this understanding may prompt athletes to find resources to manage these constructs in a convenient way.

2.3.3. Time. The relationship between competition pressure and performance is stronger, while athletes with lower skill levels have a smaller or insignificant relationship. Among high-level athletes, the presence of competitive pressure seems to improve performance. These findings are being further explored. As the skill level improves, the results of research on skill level are mixed. Compared with college athletes, the competition pressure level of elite athletes has increased. Competition pressure is most closely related to performance in the 31-59 minutes before the game. As the game time approaches, the relationship between competition pressure and performance gradually weakens.

2.3.4. Failure Appearance. Coping with stress is an important factor in the effective functioning of the sports elite, and the fear of failure (FF) is an example of the stressors experienced by athletes. Fear of failure has increased athletes' negative perceptions and concerns about failure in a short period of time. This subsequently leads to increased negative emotions (anxiety) and decreased self-awareness and motivation of athletes in the short term. The fear of failure seems to reduce the self-confidence of some athletes, make them adopt a negative attitude, and reduce their motivation to perform because they are afraid of the possibility of failure and the consequences that follow.

\section{Artificial Neural Network Model of Genetic Backpropagation}

3.1. Multilayer Feedforward Network and BP Learning Algorithm. The biggest feature of neural network is its ability to learn. In the process of learning, the connection right of the network has been changed accordingly, and the learning content has been memorized. Among them, the learning rule determines the change of the connection weight of the neural network.

The connection weight between two neurons is proportional to the activity value of the two neurons. For example, vi and vj represent the output values of the two neurons, and the change in the weight between them is

$$
\Delta w_{i j}=v_{i} \cdot v_{j} \cdot \theta
$$

where $\theta$ is the step size or constant.

As the most basic working unit of a neural network, the structure of a neuron is simple, and its processing power is relatively simple. However, a neural network composed of a large number of neurons with simple structures and functions has many superior characteristics. There are many types and different structures of artificial neural networks, which are suitable for different situations, and the difficulty of establishing and solving different types of models is also different.

The structure of the multilayer feedforward network is shown in Figure 2. Each neuron is represented by a node. The network consists of input layer, hidden layer, and output layer nodes. The hidden layer can be one layer or multiple layers, and the nodes from the front layer to the back layer are connected by weights. Because of the BP algorithm, it is often called BP neural network.

The learning process of the entire network can be divided into two stages. The first stage is to calculate from the bottom of the network. If the structure and weight of the network have been set and the known learning samples are input, the neuron output of each layer can be calculated. The second stage is to modify the weights and thresholds, which are calculations and modifications from the highest level downwards, modify the weights associated with the highest level from the known errors of the highest level, and then modify the weights of each level.

\subsection{Fusion of Backpropagation BP Neural Network and Ge-} netic Algorithm. BP network is a simple forward layered network often used in the field of neural networks. This type of neural network has the characteristics of good adaptation, self-learning, strong nonlinear approximation, large-scale parallel processing, and fault tolerance.

The network model has a hidden layer in the middle layer, uses an error propagation algorithm, and has corresponding learning rules to follow. In particular, its 
mathematical meaning is clear, and the learning algorithm with clear steps allows it to have a wider application background.

Modeling with artificial neural networks is based on the basic knowledge of biological neural network mechanisms. According to the thinking of the control process, the corresponding model is established, and appropriate algorithms are used to optimize the parameters of the network model, such as connection weights and thresholds, to obtain the optimization model of the problem. Due to the locality of the $\mathrm{BP}$ neural network, when the BP network is used to trace the source of sports competition pressure, the parameters of the obtained network model are easy to fall into the local optimum. Therefore, it is necessary to improve the defect of the BP neural network that is easy to fall into the local optimum.

On the other hand, genetic algorithms are prone to immature convergence. Although there are many corresponding improvement methods, the main purpose is to maintain the individual diversity of the algorithm, such as adjusting operating parameters and increasing the population size, without considering the improvement. The latter algorithm has learning ability and robustness, which happens to be the advantage of neural network.

It can be seen from the above that if the neural network and genetic algorithm can be combined, the advantages of both can be fully utilized, so that the new algorithm has both the learning ability and robustness of neural network and the strong global random search ability of genetic algorithm.

The combination of the two is mainly reflected in the use of genetic algorithms in neural networks, that is, to optimize the weight of the network and optimize the topology of the network. This article mainly discusses the use of genetic algorithm to optimize the weights of feedforward networks. The basic idea is to regard the learning process of the network as a process of searching for the optimal set of weights in the weight space. The system structure diagram of the combined algorithm is shown in Figure 3.

\subsection{Establishment of a Genetic Backpropagation Neural} Network Model for Stress Tracing in Sports Competitions. The basic structure of genetic backpropagation artificial neural network is forward neural network. The active state of the input unit represents the original information input into this network. The activity of each unit depends on the activity of the input unit and the connection weight between the input unit and the hidden unit. Similarly, the behavior of the output unit depends on the activity of the hidden unit and the weight between the hidden unit and the output unit. The propagation of information is from the input unit to the hidden unit and finally to the output unit. The weights between the input unit and the hidden unit and the hidden unit and the output unit determine when each unit is active. By modifying these weights, the forward neural network can be used to approximate any continuous function, which can realize the nonlinearity of the multivariate function.

For any continuous function in the closed interval, a hidden neural network can be used to approximate it. A three-layer network can complete any n-dimensional to $\mathrm{m}$-dimensional mapping, so this paper chooses a three-layer network, that is, the number of hidden layers is one layer. The selection of the number of hidden units is a very complicated problem, and there is no good analytical formula to express it. It can be said that the number of hidden units is directly related to the requirements of the problem and the number of input and output units. If the number of hidden units is too small, the network may not converge, or the network is not strong, and cannot recognize samples that have not been seen before. The fault tolerance is poor, but too many hidden units make the learning time too long and the error may not be the best. Therefore, there is an optimal number of hidden units.

$$
N^{\prime} \leq \sum_{i=1}^{n} c \cdot\left[\begin{array}{c}
i \\
n_{1}
\end{array}\right],
$$

where $k$ is the number of samples, $\mathrm{n} 1$ is the number of hidden layer units, and $n$ is the number of input units.

For the classifier, the main reason is that its input and output are basically binary functions, often the input is binary image pixels and features, and the output is 0 , 1, etc., so you can refer to the perceptron model.

In the genetic algorithm, the individuals in the initial population are randomly generated, and the determination of the population size is greatly affected by the selection operation. The larger the group size, the higher the diversity of individuals in the group, and the smaller the risk of the algorithm falling into a local solution. Therefore, starting from the group diversity, the group size should be larger. However, too large a group size will increase the amount of calculation, which will affect the efficiency of the algorithm; at the same time, when there are too many individuals in the group, a small number of highly adaptive individuals will be selected and survived, but most individuals will be eliminated, which will affect the formation of the matching library. On the other hand, the group size is too small, which will limit the distribution range of the genetic algorithm's search space, so the search may stop in the immature stage, causing immature convergence. In practical applications, the range of the number of individuals in a group is generally tens to hundreds. The population size selected in this article is 90 .

Let $a$ be the parent solution vector, and select component $a_{\mathrm{k}}$ for mutation. The children obtained after performing the mutation operation are

$$
a^{\prime}=\left(a_{1}, a_{2}, a_{3}, \ldots, a_{k}, \ldots, a_{m}\right) .
$$

Among them,

$$
a_{k}^{\prime}= \begin{cases}a_{k}-\Delta\left(b_{k}-a_{k}\right), & \operatorname{rnd}(2)=1, \\ a_{k}+\Delta\left(b_{k}-a_{k}\right), & \operatorname{rnd}(2)=0 .\end{cases}
$$

Here, rnd (2) means that the integer modulo 2 will be generated uniformly at random, and $t$ is the current evolutionary generation.

$$
\Delta(t, y)=\lambda \cdot y \cdot\left[1-r^{1-t / T}\right] .
$$




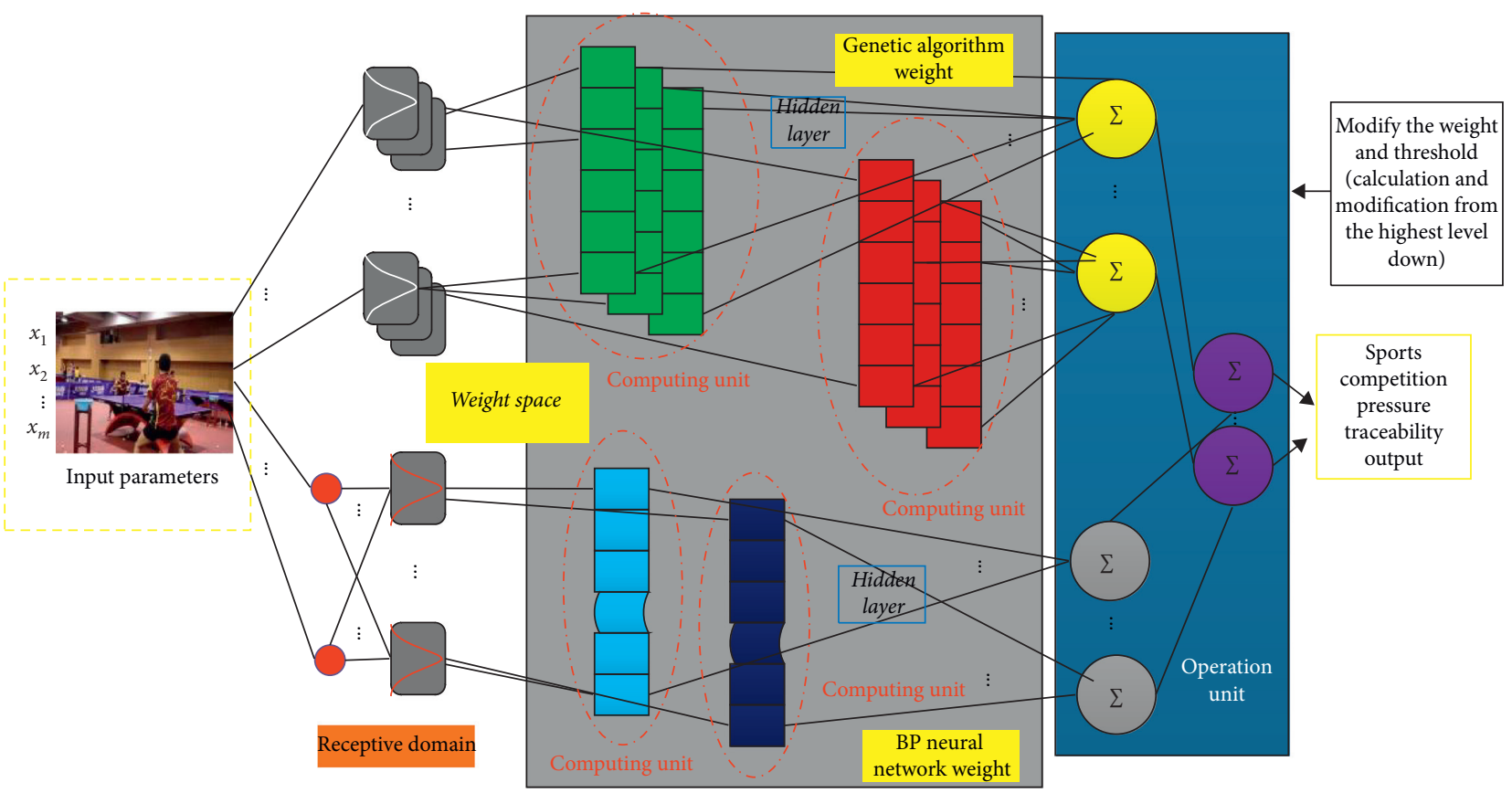

FIGURE 2: Multilayer feedforward neural network structure.

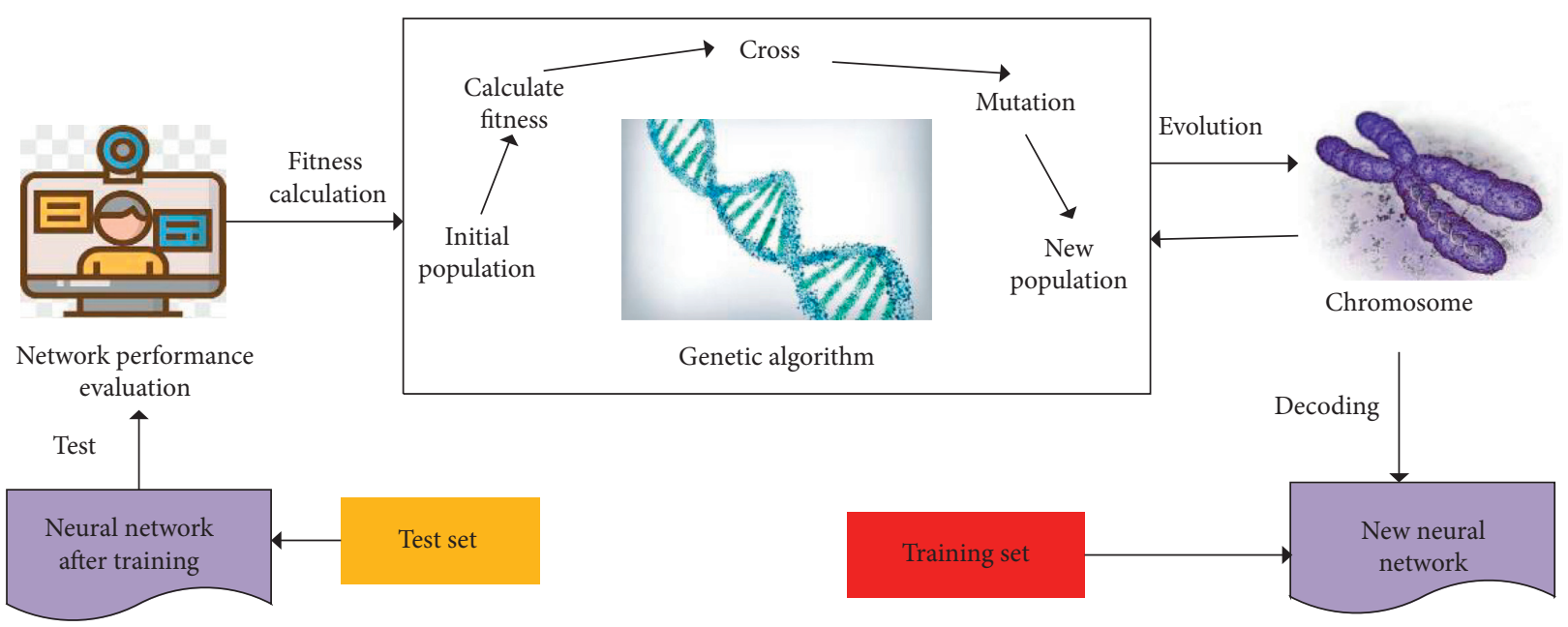

FIGURE 3: System structure diagram of GA-BP algorithm.

Among them, $r \in(0,1)$ is a random number, Trepresents the maximum algebra, and $\lambda$ is a parameter to determine the degree of inconsistency.

\subsection{The Key Algorithm and Implementation Process of the} Backward Pass GA-BP Neural Network. In this paper, genetic algorithm and BP algorithm based on gradient descent are combined to perform hybrid training on BP network. First, we use genetic algorithm to optimize the initial weight distribution, and then find the best chromosome in the optimized initial weight distribution, use BP to learn the chromosome once, and repeat this step until the accuracy requirement is met. In this way, the efficiency and effect of mixed training are better than that of using BP algorithm alone to train the network, and it can improve the convergence speed of BP network and cross the local minimum, avoiding the premature phenomenon of genetic algorithm.

When the genetic algorithm is used to optimize the initial weight distribution of the BP network, the chromosome of the genetic algorithm is the weight of the BP network, the evaluation function of the genetic algorithm is the error function of the BP network, and the satisfactory solution of the genetic algorithm is the weight vector of the BP network. The implementation process is shown in Figure 4. 


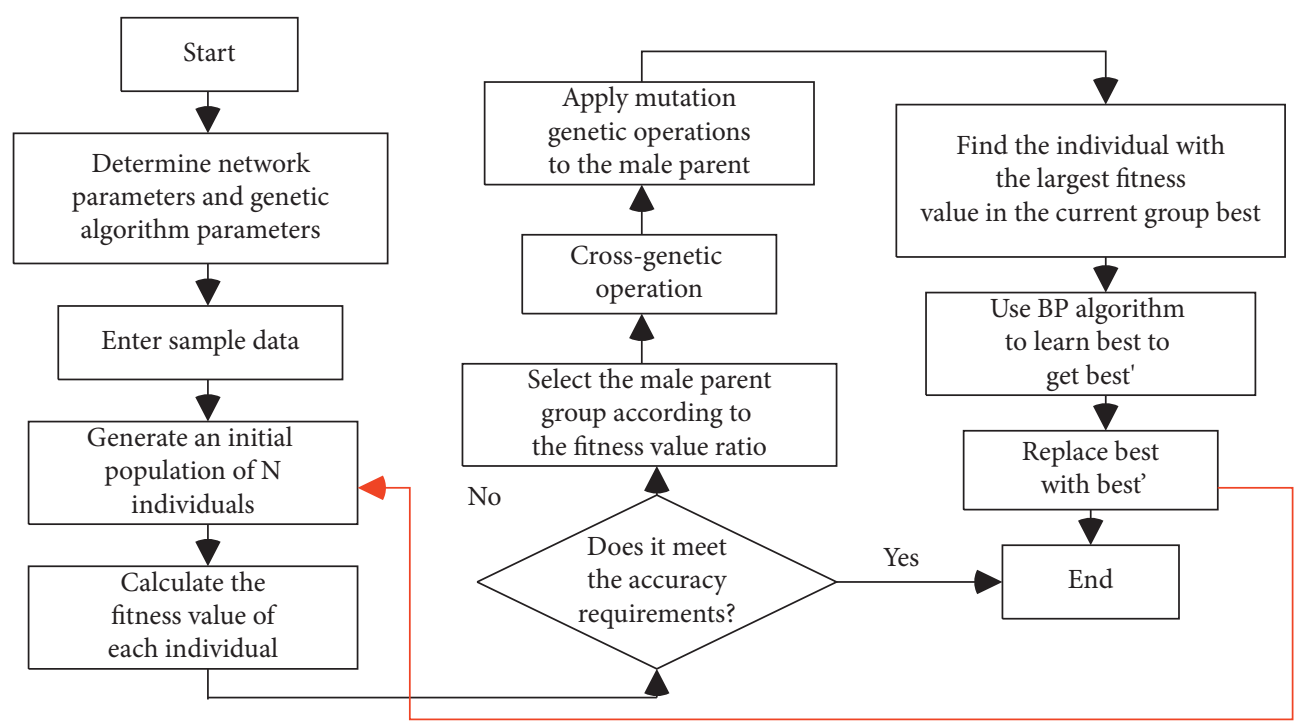

FIgURE 4: Flow chart of the backward pass GA-BP algorithm.

\section{Athlete's Competition Pressure Situation and the Analysis of the Source of the Factors Affecting Competition Pressure}

4.1. The Overall Situation of Athletes' Competition Pressure. According to the results of the questionnaire analysis during the competition of college table tennis players, we compare the average value of the various standards of the competition pressure source of each athlete, the distribution and percentage of the number of times of each event, and the statistical data of the ranking of the scale dimension scores. Research analysis shows that the overall level of competition pressure for college table tennis players is relatively high.

The researcher's questionnaire survey shows that competition pressure for college table tennis tournament athletes is widespread. The questionnaire has 35 basic items and 7 basic information items. The overall situation of competition pressure of college table tennis championship players is summarized. Under seven dimensions of competition pressure, the average score of each dimension is ranked from high to bottom. The order is competition, sports injury, and self-expectation. The corresponding average scores for studies, training, social expectations and evaluations, and interpersonal relationships are 17.751, 9.358, 8.352, 7.214, $6.566,3.914$, and 3.542. The data show that the overall stress level of athletes is relatively high, and the four dimensions of competition, sports injury, self-expectation, and academic performance have a greater impact on athletes.

It can be seen from Figure 5 that the three dimensions that have a relatively large impact on athletes' competition pressure are competition, sports injury, and self-expectation. Among them, the competition dimension scores the highest, indicating that the greatest pressure of college table tennis championship athletes comes from the competition dimension. During the entire competition process, a high degree of attention was paid to the competition, and too much consideration of competition results and tasks caused a strong psychological burden on the athletes, which in turn affected their normal performance.
Athletes are prone to psychological shadow and frustration after losing the game. Research has found that college students' table tennis tournament athletes are more troubled by opponents or spectators in the competition dimension. During onsite investigations, I often found when observing the performance of athletes on the field that when athletes score, they would shout out cheering words, and teammates would clap and applaud rhythmically. At this time, the athlete who loses points will frown and repeatedly look sideways. Therefore, in the process of competition pressure control, a combination of psychological counseling and psychological intervention should be used. Coaches should actively communicate with athletes many times, organize the competitive psychological state, and rationally view the results of the game. Secondly, sports injuries have a higher dimensional score, which ranks second, indicating that the competition pressure faced by college table tennis championship players in this competition is relatively large for sports injuries.

\subsection{Various Dimensions of Competition Pressure}

4.2.1. The Situation of the Competition Dimension. The average score of the "importance of the competition" of the college table tennis championship athletes in the competition dimension is extremely high with 3.95 points. In addition, the number of people who choose the "normal pressure," "high pressure," and "extreme pressure" options are 53,81 , and 62 , accounting for $94.3 \%$ of the total. "After losing the game," the score was 3.43 points. Among them, 100 people and 82 people were under ordinary and high pressures, accounting for $85.1 \%$. In addition, the number of people who chose the "average pressure" and "high pressure" options was 158 , accounting for $75.4 \%$ of the total. The situation of the competition dimension is shown in Figure 6.

4.2.2. The Situation of Sports Injury Dimensions. In terms of sports injury dimensions of the college table tennis 


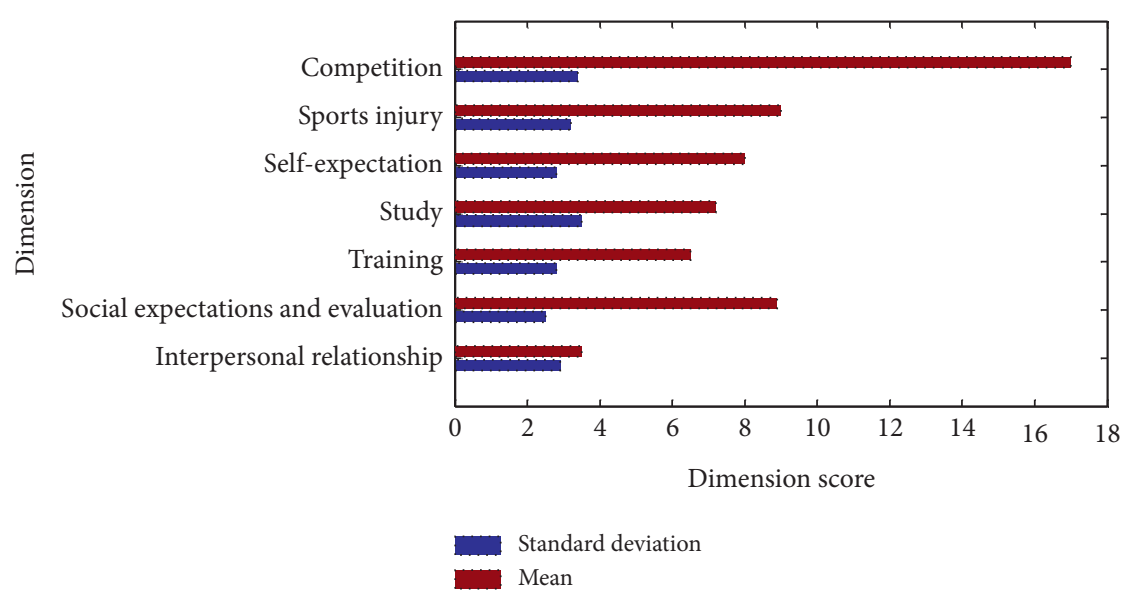

FIGURE 5: Dimensional score ranking of athletes' competition stressor scale.

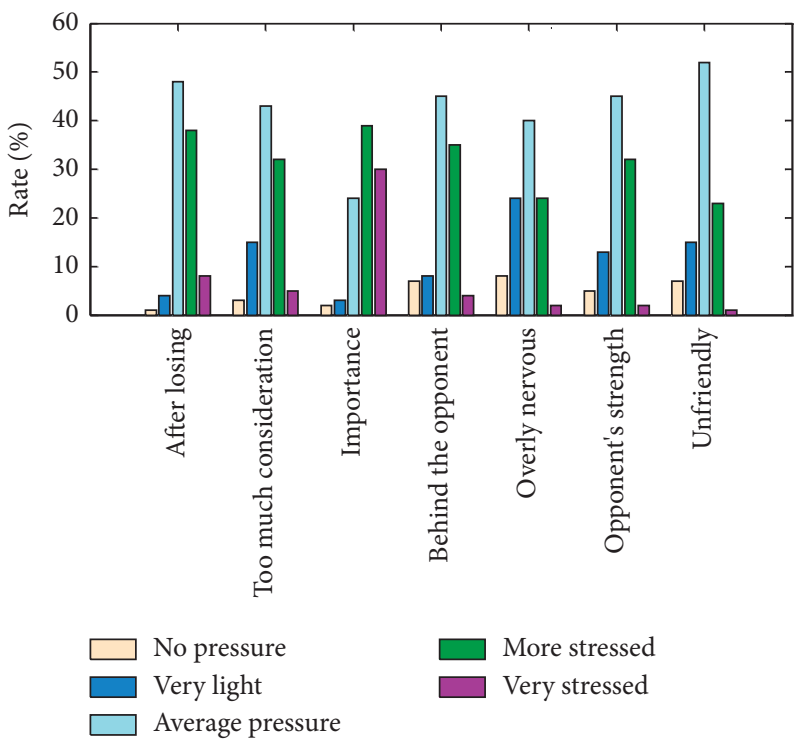

Figure 6: The situation of the competition dimension.

championship athletes, "fear of injury during training or competition," "injury during training or competition," "injuries affecting training and competition due to sports injury," scores were 3.11,3.06, and 2.82, respectively. There are 134,132 , and 135 people with more pressure options, each accounting for $64.1 \%, 62.8 \%$, and $63.4 \%$ of the total number of people. In these three items, most people are between average and high pressure. The situation of sports injury dimensions is shown in Figure 7.

4.2.3. Situation of Self-Expectation Dimension. In terms of self-expectation dimension, college table tennis tournament athletes are divided into three points: "eager to win every game," "expect to have excellent athletic performance," and "want to be the best in the team." They are 3.93, 3.32, and 2.98. Among them, 47, 21.7\%, 96, 45.1\%, 73, and $34.2 \%$ are selected under general pressure; the number and proportion of people who choose the most stressed option are $76,36.7 \%$, $26,11.9 \%, 29$, and $14.3 \%$, respectively. In these three items, most athletes are under normal pressure. The situation of self-expectation is shown in Figure 8.

In any competition, athletes strive for the highest goal and strive to win every game. Only then will they take the game seriously with fighting spirit and confidence, and "expecting oneself to have excellent athletic performance" is a demonstration and test of the results of hard training in peacetime, and a measure to fully increase one's confidence in the game and mobilize the enthusiasm of the game. "Want to be the best in the team" should be the voice of any athlete. 

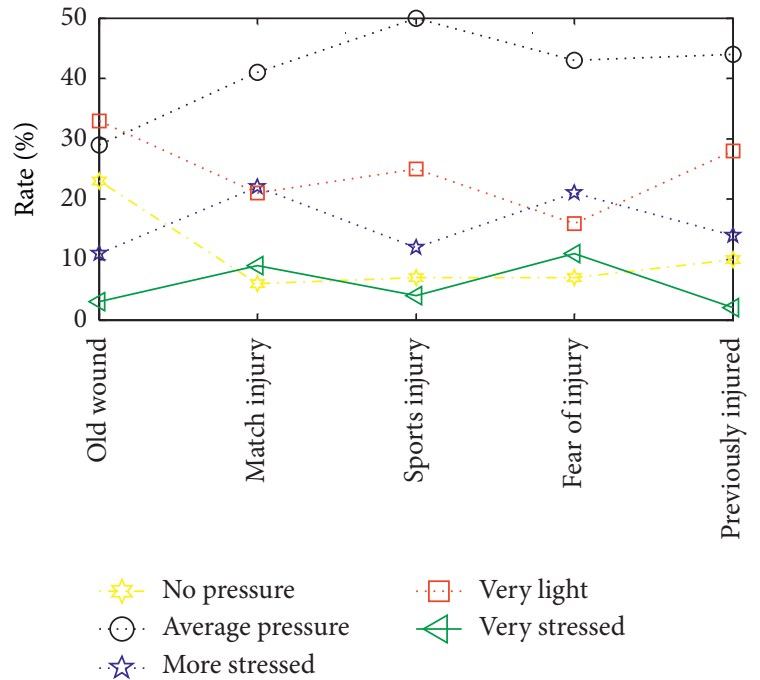

FIgURE 7: The situation of sports injury dimensions.

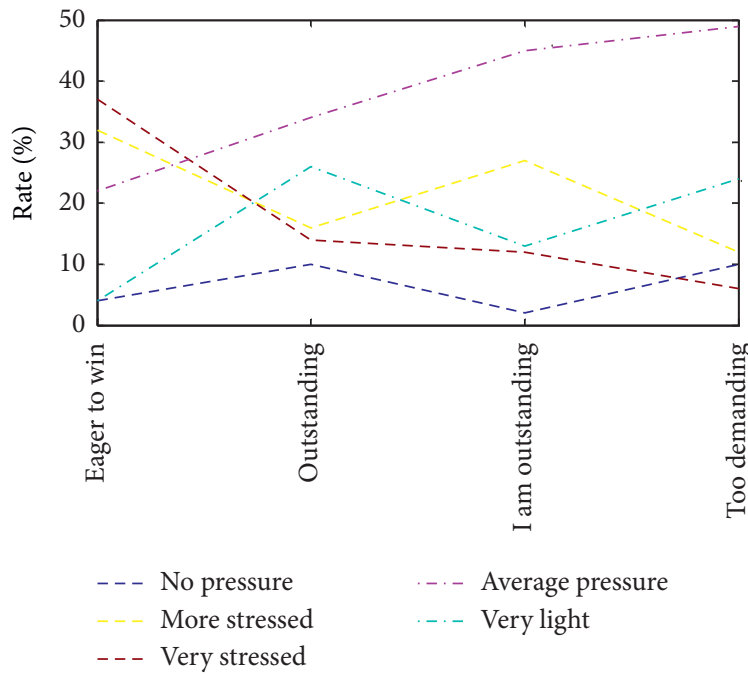

Figure 8: The situation of self-expectation.

Only when every athlete does not relax his requirements, can he have excellent skills and tactics and achieve excellent results.

\subsection{Tracing the Source of Factors Affecting Competition Pressure of College Table Tennis Championship Players}

4.3.1. The Impact of Adaptability on Athletes' Competition Pressure. As shown in Figure 9, there is a very significant difference before and after adaptation to the venue $(p<0.001)$. The pressure after adapting to the venue is significantly less than the pressure before adapting to the venue. Although for athletes, the game is not a horror movie, there will be many unknowns before adapting to the venue. After personally adapting to the venue, playing in the competition hall, and practicing with the table, you will have a great understanding of the structure of the entire venue, so that you have a bottom line and adjust in time. This is also reflected in the interviews conducted by the researchers during the athletes' adaptation period.

Adapting to the venue is very important for athletes. Before adapting to the venue, they played drums and felt a little flustered. After practicing for a while, they gradually relaxed, and the athletes noticed that the floor was slippery in advance, and at least they had an understanding of the venue in their heart, which virtually alleviated the pressure.

4.3.2. The Influence of Competition Experience Factors on Athletes' Competition Pressure. As shown in Figure 10, the surveyed athletes' experience in participating in the college table tennis tournament is divided into two criteria, one is to participate once (indicated by "first time"), and the other is to participate 2 to 8 times (because of the limitation of registration conditions).

According to the analysis of the scale and on-site interviews, most of the athletes participating in the National College Table Tennis Championships for the first time are freshmen. Such athletes, who are new to colleges, are relatively unfamiliar with national college competitions and will be more anxious in self-expectations. If you want to get results and prove yourself, for fear that your mistakes or faults will affect the team's performance, you will be more likely to have pressure in the competition. The athletes who have participated in the college table tennis championships many times are familiar with the competition system and rules and have rich experience in participating. They are even more familiar with some sports teams or some athletes. The level of participation in large-scale events has a relatively deep grasp, and the invisibly psychological pressure will be greatly reduced.

4.3.3. The Impact of Gender on Athletes' Pressure. Male and female athletes were subjected to independent sample $T$ tests, and the analysis showed that there was no significant difference in competition pressure between male and female athletes. As shown in Figure 11, there is no significant difference between male and female athletes in competition, training, school work, sports injuries, self-expectations, interpersonal relationships, social expectations, and evaluation. This is because athletes mostly start training from a young age, often participate in table tennis competitions, and are not treated differently in daily training, so there is no significant difference between male and female table tennis players in the seven dimensions of competition pressure. Athletes are a special group. Undergraduate athletes have the same set of tasks and goals, and both men and women have the same desire for competition results, so there is no obvious difference.

4.3.4. The Impact of Academic Qualifications on Athletes. According to the academic qualifications of the college table tennis tournament athletes, the subjects were divided into two levels, namely, undergraduates and graduate students. 


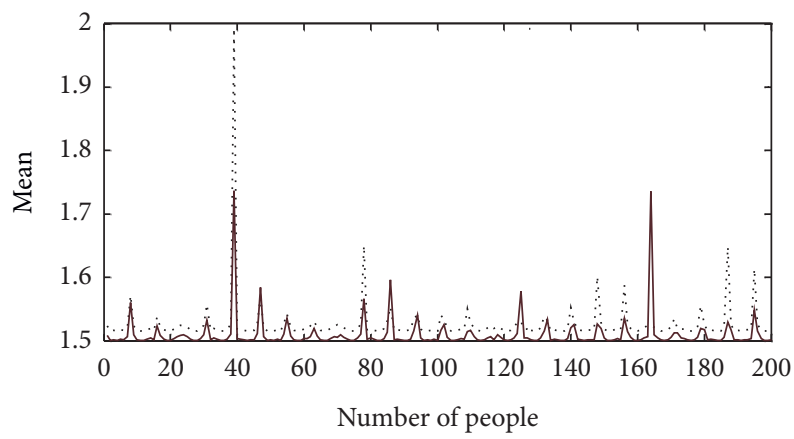

Before adapting to the venue

- After adapting to the venue

Figure 9: Analysis of differences before and after athletes adapting to the field.

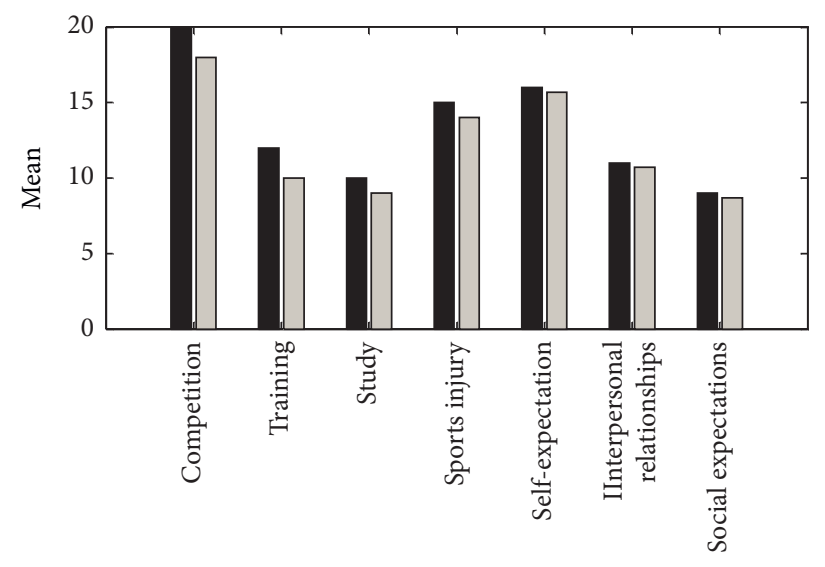

First mean

Multiple mean

Figure 10: Analysis of differences in the competition experience of college table tennis championship players.

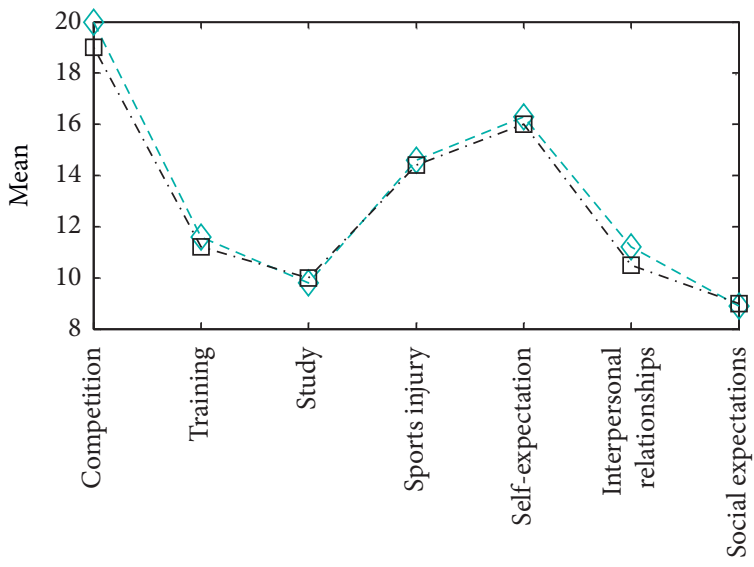

- Boys mean

Ð- Girls mean

FIGURE 11: Gender differences among table tennis players in various dimensions of competition stressors. 


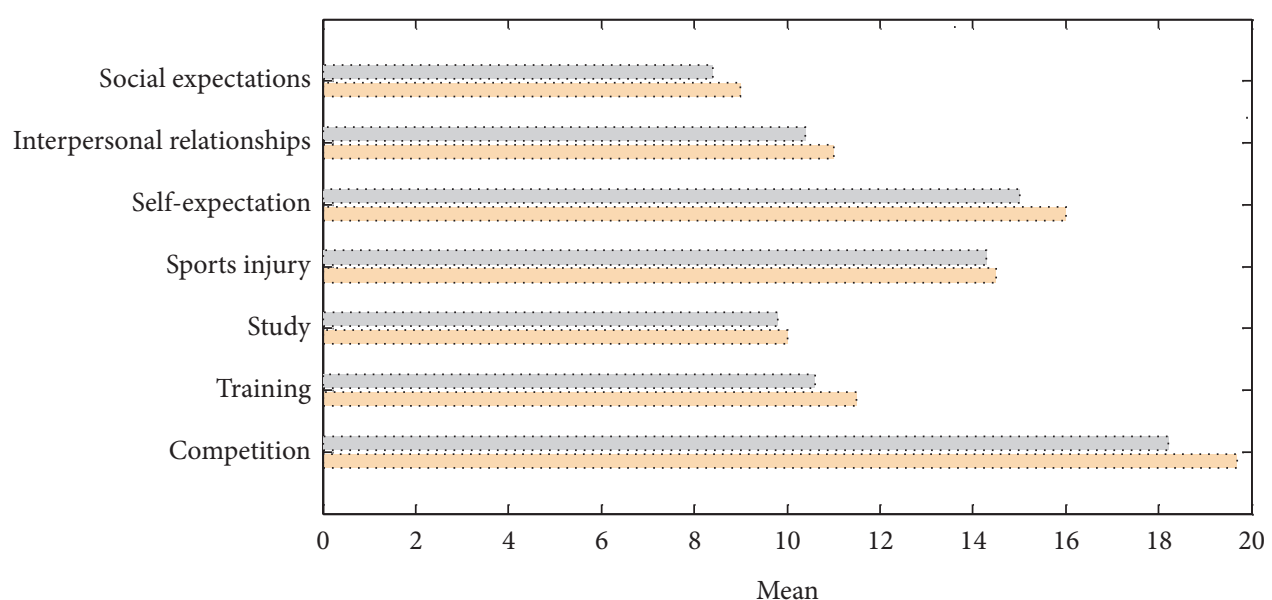

Undergraduate mean

Masters mean

FIgURE 12: Analysis of differences in athletes' academic qualifications.

The independent sample $T$ test was conducted on the two athletes with different levels of education. There are big differences in individual dimensions. As shown in Figure 12, the two groups of athletes have no significant differences in training, academic performance, sports injuries, interpersonal relationships, social expectations, and evaluation, but there are significant differences in competition and selfexpectation dimensions $(p<0.05)$. Since this year's graduates can sign up for the competition, the undergraduate group includes first-year, second-year, third-year, and fourth-year students and students who have just graduated this year. Postgraduates include first-year, second-year, third-year, and 2019 graduates.

\section{Conclusion}

From a learning point of view, GA-BP neural network is a powerful learning system with a simple system structure and easy to implement; from a system point of view, it is a static nonlinear mapping, which can be obtained through a composite mapping of simple nonlinear processing units. The BP neural network model optimized by genetic algorithm is a very effective neural network model. There are no significant differences in training, academics, sports injuries, interpersonal relationships, social expectations, and evaluations of athletes with different academic qualifications in the university table tennis championships, but there are significant differences in competition and self-expectation dimensions. Due to the particularity of the stage, the competition pressure of fourth-year undergraduate and third-year masters is significantly higher than that of the other grades. The number of athletes participating in college table tennis tournaments and their academic, sports injuries, interpersonal relationships, social expectations, and evaluation dimensions are not significant. There are very significant differences in the dimensions of competition and significant differences in the dimensions of training and selfexpectation. The competition pressure of the athletes who participated in the college table tennis championship for the first time was significantly higher than that of the athletes who participated repeatedly. There are very significant differences before and after college table tennis championship athletes adapting to the field. Before adapting to the venue, the competition pressure of the athletes is generally greater. After adapting to the venue, the competition pressure of the athletes has been relieved, indicating that it is very necessary to adapt to the venue before the competition.

\section{Data Availability}

The data used to support the findings of this study are available from the corresponding author upon request.

\section{Conflicts of Interest}

The authors declare that they have no conflicts of interest or personal relationships that could have appeared to influence the work reported in this paper.

\section{References}

[1] R. Feng, H.-j. Zheng, H. Gao et al., "Recurrent Neural Network and random forest for analysis and accurate forecast of atmospheric pollutants: a case study in Hangzhou, China," Journal of Cleaner Production, vol. 231, pp. 1005-1015, 2019.

[2] T. Li and J. Song, "Research on promotion methods of positive mental health of college students under the model of ecological sports teaching," Ekoloji, vol. 28, no. 107, pp. 18611868, 2019.

[3] M. Da Lio, D. Bortoluzzi, and G. P. Rosati Papini, "Modelling longitudinal vehicle dynamics with neural networks," Vehicle System Dynamics, vol. 58, no. 11, pp. 1675-1693, 2020.

[4] C. Wang, D. Li, Z. Li et al., "An efficient local binary pattern based plantar pressure optical sensor image classification using convolutional neural networks," Optik, vol. 185, pp. 543-557, 2019. 
[5] H. Ma and X. Pang, "Research and analysis of sport medical data processing algorithms based on deep learning and internet of things," IEEE Access, vol. 7, pp. 118839-118849, 2019.

[6] A. Falamarzi, S. Moridpour, M. Nazem, and S. Cheraghi, "Prediction of tram track gauge deviation using artificial neural network and support vector regression," Australian Journal of Civil Engineering, vol. 17, no. 1, pp. 63-71, 2019.

[7] A. Bonfitto, A. Tonoli, S. Feraco, E. C. Zenerino, and R. Galluzzi, "Pattern recognition neural classifier for fall detection in rock climbing," Proceedings of the Institution of Mechanical Engineers, Part P: Journal of Sports Engineering and Technology, vol. 233, no. 4, pp. 478-488, 2019.

[8] D. Patel, D. Shah, and M. Shah, "The intertwine of brain and body: a quantitative analysis on how big data influences the system of sports," Annals of Data Science, vol. 7, no. 1, pp. 1-16, 2020.

[9] R. Donald, T. Howells, T. Howells et al., "Forewarning of hypotensive events using a Bayesian artificial neural network in neurocritical care," Journal of Clinical Monitoring and Computing, vol. 33, no. 1, pp. 39-51, 2019.

[10] J. F. Dunne and C. Bennett, "A crank-kinematics-based engine cylinder pressure reconstruction model," International Journal of Engine Research, vol. 21, no. 7, pp. 1147-1161, 2020.

[11] E. Finnegan, M. Villarroel, C. Velardo, and L. Tarassenko, "Automated method for detecting and reading seven-segment digits from images of blood glucose metres and blood pressure monitors," Journal of Medical Engineering \& Technology, vol. 43, no. 6, pp. 341-355, 2019.

[12] P. R. Duchowicz, "QSPR studies on water solubility, octanolwater partition coefficient and vapour pressure of pesticides," SAR and QSAR in Environmental Research, vol. 31, no. 2, pp. 135-148, 2020.

[13] H. Yu, N. Ji, Y. Ren, and C. Yang, “A special event-based K-nearest neighbor model for short-term traffic state prediction," IEEE Access, vol. 7, pp. 81717-81729, 2019.

[14] X. Shen, Y. Zheng, and R. Zhang, "A hybrid forecasting model for the velocity of hybrid robotic fish based on back-propagation neural network with genetic algorithm optimization," IEEE Access, vol. 8, pp. 111731-111741, 2020.

[15] H. Ba, "Medical sports rehabilitation deep learning system of sports injury based on MRI image analysis," Journal of Medical Imaging and Health Informatics, vol. 10, no. 5, pp. 1091-1097, 2020.

[16] A. Sharma, Y. Singh, and N. A. Kumar, "Effect of fuel injection pressure and timing on Polanga (Calophyllum Inophyllum) biodiesel blends for engine performance and emissions analysis," Energy Sources, Part A: Recovery, Utilization, and Environmental Effects, vol. 41, no. 24, pp. 3046-3057, 2019.

[17] X. Schelling and S. Robertson, "A development framework for decision support systems in high-performance sport," International Journal of Computer Science in Sport, vol. 19, no. 1, pp. 1-23, 2020.

[18] M. Campo, S. Champely, B. Louvet et al., "Group-based emotions: evidence for emotion-performance relationships in team sports," Research Quarterly for Exercise and Sport, vol. 90, no. 1, pp. 54-63, 2019.

[19] C. L. Atkinson, "Full and open competition in public procurement: values and ethics in contracting opportunity," International Journal of Public Administration, vol. 43, no. 13, pp. 1169-1182, 2020.

[20] D. Ilić and V. Mrdaković, "How to win a medal in individual sports in three consequtive Olympic cycles," Fizička Kultura, vol. 73, no. 1, pp. 106-119, 2019.
[21] T. Nandi, R. Lewthwaite, B. E. Fisher, and G. J. Salem, "Balance confidence scale: preliminary validity, reliability, and relation to neural excitability in young adults," Psychology of Sport and Exercise, vol. 43, pp. 301-310, 2019.

[22] S. Credé, T. Thrash, C. Hölscher, and S. I. Fabrikant, "The acquisition of survey knowledge for local and global landmark configurations under time pressure," Spatial Cognition \& Computation, vol. 19, no. 3, pp. 190-219, 2019.

[23] H. Hu, B. Tang, X. Gong, W. Wei, and H. Wang, "Intelligent fault diagnosis of the high-speed train with big data based on deep neural networks," IEEE Transactions on Industrial Informatics, vol. 13, no. 4, pp. 2106-2116, 2017.

[24] W. Wu, X. Xia, M. Wozniak, X. Fan, R. Damaševičius, and Y. Li, "Multi-sink distributed power control algorithm for Cyber-physical-systems in coal mine tunnels," Computer Networks, vol. 161, pp. 210-219, 2019.

[25] D. Połap, M. Woźniak, W. Wei et al., "Multi-threaded learning control mechanism for neural networks," Future Generation Computer Systems, vol. 87, pp. 16-34, 2018. 